\title{
Colestasia Intrahepática Familiar Progresiva Tipo 3: Presentación de Casos Clínicos y Actualización de Tema
}

\author{
JOSÉ ZACARÍAS S. ${ }^{1}$ \\ 1. Profesor Titular Universidad de Chile, Unidad de Gastroenterología Clínica Las Condes.
}

\begin{abstract}
Type 3 Familial Intrahepatic Cholestasis: Clinical Cases and Update

Abstract: Familial Intrahepatic Cholestasis (FIC) includes a heterogeneous group of recessive autosomic alterations characterized by hepatocellular cholestasis secondary to the interruption of the normal process of synthesis of bilis. Objective: A description of FIC in 3 of 5 children of an index family. Clinical case: a 5 y.o. child with hepatosplenomegaly increased serum hepatic enzymes and biliary acids. Abdominal echography showed alterations compatible with hepatic fibrosis. Biopsy showed bridge fibrosis, duct proliferation, minimal chronic cholestasis. These findings were compatible with a phenotype FIC-3 with elevate levels of Gamma-glutamyl transferase. A mutation of MDR3 gene is responsible for the absence of biliary phospholipids, allowing a detergent effect of biliary acids upon the duct epithelium, developing cholangitis, fibrosis and later cirrhosis. Among four brothers, the mutation was found in two twin sisters. Three affected brothers were treated with ursodeoxicolic acid, $30 \mathrm{mg} / \mathrm{Kg}$. Excellent results were obtained in the twin girls not in the index boy. The clinical expression of this illness is variable, and an elevation of aminotransferase must call attention to this possibility. Early diagnostic and treatment could avoid the development of hepatic damage and cirrhosis.
\end{abstract}

(Key words: Intrahepatic cholestasis, familial cholestasis, type 3).

Rev Chil Pediatr 2009; 80 (5): 459-466

\section{RESUMEN}

La Colestasia Intrahepática Familiar Progresiva (CIFP) comprende un grupo heterogéneo de alteraciones autosómicas recesiva caracterizadas por una colestasia hepatocelular secundaria a una interrupción del proceso normal de síntesis de la bilis. Objetivo: Describir la presentación de CIFP en 3 de 5 hijos de una familia estudiada. Caso clínico: Paciente de 5 años de edad (caso 1), que presenta una hepatoesplenomegalia,

Trabajo recibido el 06 de abril de 2009, devuelto para corregir el 17 de junio de 2009, segunda versión el 22 de junio de 2009, aceptado para publicación el 02 de julio de 2009.

Correspondencia a:

José Zacarías S.

drzacarias@hotmail.com 
aumento de enzimas hepáticas y de ácidos biliares en suero. La ecotomografía abdominal describe alteraciones compatibles con fibrosis hepática. La biopsia reveló fibrosis en puente, proliferación ductular y colestasia crónica mínima. Estos hallazgos fueron compatibles con el fenotipo de una CIFP-3 con niveles elevados de Gamaglutamiltransferasa (GGT). Una mutación del gen MDR3 es responsable de la ausencia de fosfolípidos en la bilis, lo que permite la acción detergente de los ácidos biliares sobre el epitelio de los conductos desencadenando una colangitis, fibrosis y luego cirrosis. De los cuatro hermanos del caso 1 se detectó la enfermedad en 2 hermanas gemelas (casos 2 y 3). Estos tres niños afectados fueron tratados con ácido ursodeoxicólico $30 \mathrm{mg} / \mathrm{kilo} /$ peso, logrando excelentes resultados en las gemelas pero no en el caso 1 . Conclusión: Se presenta a 3 hermanos con el fenotipo de CIFP. La expresión clínica de esta enfermedad puede ser variable y de manifestación tardía, la elevación de las aminotransferasas debe considerar esta patología en el diagnóstico diferencial de las numerosas causas que dan origen a un aumento de estas enzimas. Sólo el diagnóstico y tratamiento precoz puede evitar la evolución a un daño hepático irreversible como es la cirrosis.

(Palabras clave: Colestasia intrahepática, familiar, tipo 3).

Rev Chil Pediatr 2009; 80 (5): 459-466

\section{Introducción}

La Colestasia Intrahepática Familiar Progresiva (CIFP) comprende un grupo heterogéneo de alteraciones autosómicas recesivas de los niños que se manifiesta por un impedimento en el proceso de síntesis de la bilis normal y se presenta como una colestasia de origen hepatocelular. Su prevalencia en la población varía entre $1 / 50000$ a 1/100 000. Se han descrito tres tipos de CIFP relacionadas a mutaciones de los genes que codifican las proteínas involucradas en los procesos de transporte de los diferentes componentes de bilis (tabla 1). La CIFP-1 y 2 se presentan en el primer mes de vida a diferencia de la CIFP-3 que se manifiesta con síntomas en etapas más avanzada de la vida (escolar, adolescente o adulto joven), a pesar de que pueden detectarse alteraciones bioquímicas desde el primer año de vida y alteraciones genéticas desde el nacimiento. Los pacientes con CIFP-3 desarrollan fibrosis hepática que progresa a cirrosis antes de la segunda década de la vida. Tanto la CIFP-1 y 2 son provocadas por una insuficiente secreción de sales biliares debido a un defecto en el gen

Tabla 1. Colestasia intrahepática familiar no obstructiva

\begin{tabular}{|c|c|c|c|c|c|}
\hline Nombre & Variedades & Defecto & Cromosoma & Fenotipo & Tratamiento \\
\hline \multirow[t]{2}{*}{ CIFP - 1} & - Enf. de Byler & $\begin{array}{l}\text { Mutación en gen } \\
\text { FIC1 (ATB8B1) (2) }\end{array}$ & & $\begin{array}{l}\text { Colestasia progresiva } \\
\text { GGT normal }\end{array}$ & $\begin{array}{l}\text { Ácido ursodeoxicólico } \\
\text { Trasplante }\end{array}$ \\
\hline & - CIRB 1 & $\begin{array}{l}\text { Mutación tipo } \\
\text { missence leve }\end{array}$ & $18 q \quad 21-22$ & $\begin{array}{l}\text { Colestasia recurrente } \\
\text { GGT normal }\end{array}$ & Acido ursodeoxicólico \\
\hline \multirow[t]{2}{*}{ CIFP - 2} & - Sínd. de Byler (5) & $\begin{array}{l}\text { Mutación de BESB } \\
\text { (ABCB11) }\end{array}$ & & $\begin{array}{l}\text { Colestasia permanente } \\
\text { GGT normal }\end{array}$ & $\begin{array}{l}\text { Ácido ursodeoxicólico } \\
\text { Trasplante }\end{array}$ \\
\hline & - CIRB 2 & $\begin{array}{l}\text { Mutación tipo } \\
\text { missence leve }\end{array}$ & $2 q-24$ & $\begin{array}{l}\text { Colestasia recurrente } \\
\text { GGT normal }\end{array}$ & Ácido ursodeoxicólico \\
\hline CIFP - 3 & & $\begin{array}{l}\text { Mutación de } \\
\text { MDR3 (4) (ABCB4) }\end{array}$ & $7 q-21$ & $\begin{array}{l}\text { Colestasia progresiva } \\
\text { GGT aumentada }\end{array}$ & $\begin{array}{l}\text { Ácido ursodeoxicólico } \\
50 \% \text { recuperación } \\
\text { Trasplante }\end{array}$ \\
\hline
\end{tabular}

(1) CIFP = Colestasia intrahepática familiar progresiva. (2) Nueva denominación de los genes. (3) CIRB = Colestasia intrahepática recurrente benigna. (4) Mutación de penetración variable. (5) Síndrome de Byler: no descendiente de familia Byler. 
ATB8B1 que codifica la proteína FIC1 y del gen ABCB11 que codifica la proteína excretora de sales biliares (BSEP) $)^{1,2}$. En el caso de CIFP-3, la mutación corresponde a la del gen ABCB4 que codifica a la proteína "multydrug resistant 3" (MDR3) que es la encargada de transportar la fosfadiatilcolina desde los hepatocitos al epitelio de las vías biliares desde donde son extraídas por las sales biliares ${ }^{3}$. Estos fosfolípidos en la bilis neutralizan la acción detergente de los ácidos biliares. La mutación de MDR3 si es del tipo de gen truncado no transporta fosfolípidos, si la mutación es del tipo "missense" transporta una mínima cantidad que permite una respuesta al tratamiento. La ausencia de fosfolípidos en la bilis es la causa por lo cual las sales biliares dañan el epitelio de los conductos desencadenando una colangitis que progresa a fibrosis hepática y luego a cirrosis.

Se reconoce actualmente CIFP-3 como una enfermedad genética localizada en el cromosoma 7 q21, en el cual se identifica una mutación parcial o total del gen que comanda la actividad del transportador MDR $3^{4}$.

En esta publicación se presenta una familia de cinco hijos, tres de los cuales presentan el fenotipo de CIFP-3. Se describe la patogenia, alteraciones clínicas, de laboratorio y resultado del tratamiento en 3 de los hermanos afectados.

\section{Casos Clínicos}

\section{Caso 1}

Niño de 5 años y 2 meses de edad, que consulta a su pediatra por una dermatitis pruriginosa de codos y rodillas. Se indicó aplicar un ungüento dérmico en la región afectada y se solicitó un perfil bioquímico. En este examen se detectó elevación de la aspartatoaminotransferasa (AST) de $187 \mathrm{UI} / 1$ y el resto de los exámenes fueron normales. Fue derivado a un pediatra gastroenterólogo en Santiago, quién describe un niño en buenas condiciones generales y nutricionales, sin ictericia, y que al examen segmentario detectó una hepatomegalia a $3 \mathrm{~cm}$ bajo el reborde costal de consistencia aumentada, sin esplenomegalia ni circulación venosa superficial abdominal. Tampoco observó estigmas de daño hepático crónico. Solicitó exámenes: AST $82 \mathrm{U} / \mathrm{L}$, alaninoaminotransferasa (ALT) $169 \mathrm{U} / \mathrm{L}$, gamaglutamiltransferasa (GGT) 442 U/L, alfa-1-antitripsina normal, panel de virus de hepatitis negativo, ceruloplasmina normal, anticuerpo antinuclear $(+) 1 / 320$ a 1/160, y anticuerpo antimitocondrial negativo. En la ecotomografía abdominal el hígado se observó aumentado de tamaño y presentó estructura heterogénea, sin dilatación de la vía biliar intrahepática, la vesícula biliar aparece colapsada, sin evidencias de litiasis. la vena porta fue descrita como prominente, con un diámetro aproximado de 7,9 $\mathrm{mm}$. La vía biliar de aspecto normal. Páncreas normal. El bazo se observó aumentado de tamaño, con un diámetro coronal de $11,2 \mathrm{~cm}$, estructura homogénea. Riñones normales. No se observó ascitis ni masas intraabdominales. La ecografía concluye una hepatomegalia heterogénea y una esplenomegalia homogénea.

Se solicitó endoscopia digestiva alta que reveló pequeñas várices esofágicas en el tercio distal del esófago. La biopsia hepática confirmó una fibrosis hepática con formación de puentes fibrosos en algunas áreas, alteración degenerativa de los conductos biliares, proliferación ductular periférica, colestasia crónica mínima e infiltración linfocitaria portal leve con lámina limitante normal. Reacción de PAS con fuerte positividad citoplasmática, la cuál se negativizó en presencia de diastasa (glicógeno). La tinción de Pearls $(\mathrm{Fe}+)$ fue negativa. La tinción de Schikata fue positiva débil, concluyendo un daño hepático crónico con fibrosis en puente y colestasia crónica leve.

En vista de la evolución asintomática, se decidió estudiar a sus 4 hermanos, dos de los cuáles (casos 2 y 3 ) presentaron niveles elevados de aminotransferasas, de GGT y fibrosis hepática (por ecotomografía). El hermano menor nació en Junio de 2006, hasta el momento (Marzo del 2009) está sano y continúa con exámenes periódicos de control. La hermana mayor de 11 años de edad, está sana y continúa con controles periódicos.

\section{Caso 2}

La niña de 9 años 6 meses (gemela 1), con un peso $25 \mathrm{kilos}$, talla 1,26 1/2 mt (talla: percentil 8 ), se encontraba en buenas condiciones gene- 
rales, sin manifestaciones clínicas de daño hepático, excepto por hígado palpable a $2 \mathrm{~cm}$ del reborde costal, de consistencia aumentada y proyección de $8 \mathrm{~cm}$. Exámenes solicitados: AST 119 U/1, LDH 778 y GGT 409 U/L, bilirrubinemia total $0,97 \mathrm{mg} / \mathrm{dl}$, y anticuerpos antinucleares negativo. La ecotomografía abdominal mostró un hígado aumentado de tamaño, de grado leve a moderado, llamó la atención la heterogeneidad del parénquima, que lo comprometía en forma difusa. No había dilatación de la vía biliar intrahepática ni masas de sustitución. Vesícula biliar de paredes finas y contenido homogéneo, sin evidencias de litiasis. Páncreas normal. Bazo midió aproximadamente $9,5 \mathrm{~cm}$, normal para la edad, estructura homogénea. La endoscopia de esófago, estómago y duodeno fue normal, la biopsia de duodeno fue normal y la biopsia hepática mostró tejido hepático con arquitectura parcialmente distorsionada, muestra fragmentada, que incluyó algunos espacios porta conservados. Presentó disminución de ductus y con estrechamiento acentuado del lumen y fibrosis. Desarrollo de septos fibrosos, que en partes alcanzan la vena central. Proliferación de tejido elástico y presencia de cordones epiteliales (ductus remanentes). Infiltrado inflamatorio linfocitario leve. No se evidencia necrosis, granulomas ni acúmulos linfoides. Parénquima, lobulillos conservados sin colestasia hepatocitaria ni canalicular. No se reconocen inclusiones virales ni presencia de depósitos de cobre. La antitransglutaminasa resultó normal $(1,8 \mathrm{EU} / \mathrm{ml})$.

\section{Caso 3}

De 9 años y 6 meses (gemela 2), con un peso de $25,2 \mathrm{~kg}$ y una talla de $1,27 \mathrm{~m}$, la niña presentaba dolor abdominal ocasional. Al examen se describe en buen estado general y nutricional, sin ictericia ni estigma de daño hepático crónico. El examen físico segmentario fue negativo, excepto en el abdomen, donde se palpó el hígado a $1 \frac{1}{2} \mathrm{~cm}$ bajo el reborde costal, de consistencia algo aumentada, proyección de $7 \mathrm{~cm}$. Los exámenes revelaron aumento de las aminotransferasas, de la GGT y anticuerpos antinucleares positivos $1 / 80$, IgA sérica 200 $\mathrm{mg} / \mathrm{dl}$, anticuerpo antitransglutaminasa 3,5 EU/ $\mathrm{ml}($ normal $<20 \mathrm{EU} / \mathrm{ml})$.
La ecotomografía abdominal mostró un hígado aumentado de tamaño, de grado leve, y su estructura se apreció heterogénea, con discreto aumento de la ecogenicidad periportal, sin evidencias de dilatación de vía biliar intrahepática ni lesiones de sustitución. Vesícula biliar distendida, de paredes lisas y contenido homogéneo, sin evidencias de litiasis. La biopsia hepática mostró un tejido hepático de arquitectura distorsionada, muestra fragmentada con espacios porta conservados. Presentaba disminución acentuada de ductus hepático, fibrosis densa, en partes hialinización peri-ductal con atrofia epitelial y áreas cicatriciales con obliteración del lumen. Desarrollo de algunos puentes fibrosis con proliferación de tejido elástico y presencia de algunos cordones epiteliales (pequeños ductus) remanente. Infiltrado linfocitario leve, parénquima lobulillar conservado, disminución de ductus interlobulillares. No se evidenció colestasia hepatocitaria ni canalicular, no se observaron depósitos de cobre ni inclusiones virales. No se observó necrosis, granulomas ni acúmulos linfoides.

Con los resultados de los exámenes de laboratorio y biopsia hepática (tabla 2), se plantearon las siguientes posibilidades diagnósticas en los 3 hermanos:

1. Enfermedad colestásica crónica genética.

2. Colangitis esclerosante.

Los padres deciden optar por otra opinión, por lo cual viajaron con el caso 1 a EE.UU. al Children's Hospital de Cincinnatti. Allá se realizó una colangiografía retrógrada endoscópica que reveló vías biliares normales y vesícula biliar aumentada de tamaño, lo que descartó una colangitis esclerosante. En la endoscopia del tubo digestivo no se encontró várices esofágicas pero sí pequeñas erosiones en el duodeno y yeyuno. Se tomaron biopsias de la zona afectada, el estudio histológico mostró una inflamación aguda de la lámina propia, algunos neutrófilos en las criptas y vellosidades intestinales normales. En los exámenes de laboratorio se encontró aumento de los ácidos biliares en el suero (19,0 U/ml/L) (rango normal: 0-6), déficit de $\operatorname{IgA}$ sérica $(<6 \mathrm{mg} / \mathrm{dl})$, anticuerpos antinucleares positivos (1:320 a 1:160) y los antígenos de histocompatibilidad HLA DQ $2(+)$ y HLA 
Tabla 2. Tratamiento con ácido ursodeoxicólico

\begin{tabular}{|c|c|c|c|c|c|c|c|c|c|c|c|}
\hline \multicolumn{12}{|c|}{ CASO 1 Masc. (Edad 5 a. 2 m.) } \\
\hline & Dic.05 & Feb.06 & Mayo & Agosto & Oct. & Nov.06 & Feb.07 & Junio & Sept. & Mar. 08 & Jul. 08 \\
\hline AST u/L & 82 & 122 & 101 & 137 & 103 & Trat. & 85 & 71 & 32 & 67 & 62 \\
\hline ALT u/L & 169 & 152 & 138 & 197 & 126 & URSO & 100 & 78 & 36 & 60 & 59 \\
\hline GGT u/L & 442 & 306 & 420 & - & 274 & & 160 & 119 & 110 & 85 & 63 \\
\hline
\end{tabular}

\begin{tabular}{lccccccccc}
\hline & \multicolumn{10}{c}{ CASO 2 Fem. (Edad 9 a. 6 m.) } & & \\
& Enero 06 & Feb. & Abril & Junio & Oct. & Nov.06 & Feb.07 & Sept. & Julio 08 \\
\hline AST u/L & 501 & 117 & & 93 & 177 & Trat. & 54 & 32 & 23 \\
ALT u/L & 522 & 91 & 119 & 72 & 242 & URSO & 48 & 34 & 30 \\
GGT u/L & 452 & 297 & & 370 & 518 & & 84 & 25 & 23 \\
Bil.T mg/dl & 1,01 & & 0,97 & & & & & & \\
\hline
\end{tabular}

\begin{tabular}{lcrrrrrrr}
\hline & & \multicolumn{7}{c}{ CASO $\mathbf{3}$ Fem. (Edad 9 a. 6 m.) } \\
& Enero 06 & Feb. & Junio & Oct. & Nov.06 & Feb.07 & Junio & Julio \\
\hline AST u/L & 473 & 125 & 62 & 193 & Trat. & 40 & 28 & 14 \\
ALT u/L & 493 & 81 & 55 & 117 & URSO & 45 & 35 & 28 \\
GGT u/L & 332 & 271 & 254 & 509 & & 37 & 8 & 14 \\
Bil.T mg/dl & & 0,26 & 0,71 & & & & & 1,33 \\
\hline
\end{tabular}

DQ 8 (-). Estos antígenos son positivos en el $37 \%$ de la población general de EE.UU. y en el 96 al 99\% de los enfermos celíacos. Se descartó el diagnóstico de enfermedad celíaca en los 3 hermanos, por presentar antitransglutaminasa normal y biopsia intestinal que reveló presencia de vellosidades intestinales normales. El caso 1 es portador de un déficit de IgA sérica, por lo que la antitransglutaminasa IgA resultó normal y la biopsia duodenal fue normal. Se descartó además el diagnóstico de enfermedad inflamatoria crónica y daño hepático secundario, para lo cuál se solicitó cintigrafía de intestino delgado con leucocitos marcados que resultó negativo, igualmente los anticuerpos antisacaromises IgA e IgG fueron negativos. Los estudios serológicos de enfermedad celíaca, antitransglutaminasa en los casos 2 y 3 , que no tienen déficit de $\operatorname{IgA}$ resultaron normales y la biopsia intestinal demostró la presencia de vellosidades intestinales normales, descartando la posibilidad de este diagnóstico. Se descartó además el diagnóstico de enfermedad inflamatoria crónica y daño hepático secundario, para lo cuál se solicitó cintigrafía de intestino con leucocitos marcados y que resultó negativo, igualmente, los anticuerpos antisacaromises IgA e IgG fueron negativos.
En Noviembre del 2006, los 3 hermanos continuaban con sus exámenes bioquímicos alterados y habiendo ya descartado los siguientes diagnósticos: colangitis esclerosante, enfermedad de Wilson, fibrosis hepática congénita, deficiencia de fosfomanosa isomerasa (fibrosis hepática por deficiencia de la N-Glicosilasion hepática), que se descartó con el examen de "Isoelectric focusing de transferrina sérica", que se realizó en Bruselas.

Finalmente, se concluyó que el síndrome colestásico no obstructivo de los 3 hermanos afectados, era compatible con CIFP-3, en la cuál existe una mutación genética de la proteína transportadora de fosfolípidos (MDR3). De acuerdo con la experiencia de otros autores ${ }^{5,6}$ se inició tratamiento con ácido ursodeoxicólico $30 \mathrm{mg} / \mathrm{kg}$ peso/día.

\section{Evolución}

Al inicio del tratamiento con ácido ursodeoxicólico, los 3 hermanos seguían asintomáticos, sólo era evidente a la palpación, el aumento de tamaño y consistencia del hígado, además de la alteración de las aminotransferasas y de las GGT. El resto del examen físico era 
negativo. Se realizaron pruebas funcionales hepáticas en forma periódica, demostrando una notoria tendencia a la normalización en los casos 2 y 3 (hermanas gemelas), pero sólo una disminución parcial de las alteraciones de las pruebas funcionales hepáticas en el caso 1 (tabla 2). Esta diferencia de respuesta se podría deber a que la mutación genética del caso 1 es probablemente completa (gen truncado) a diferencia de sus hermanas, que debe ser sólo parcial (missense).

\section{Discusión}

Al revisar los síndromes colestásicos genéticos en niños, encontramos la descripción de 3 diferentes enfermedades colestásicas no obstructivas (tabla 1). La CIFP, de las cuáles la más conocida por los pediatras es la CIFP-1, llamada enfermedad de Byler, se manifiesta desde el primer mes de vida con ictericia, posteriormente prurito, diarrea, retraso del desarrollo y finalmente cirrosis hepática ${ }^{7}$. Gracias a la técnica quirúrgica que desvía la secreción biliar hacia el exterior, llevando el colédoco hacia una ostomía en la pared abdominal, evitando la reabsorción de las sales biliares, se ha logrado atenuar los síntomas y progresión del daño hepático, logrando así alcanzar una edad mayor con el fin de realizar un trasplante ${ }^{8,9}$.

De las tres CIFP descritas, sólo una evoluciona con aumento de la GGT, es la CIFP-3. En estos enfermos se ha confirmado una mutación parcial o total del gen de la lipoproteína denominada multidrug resistant-3, que se localiza en la pared de los conductos biliares y cuya acción es transportar la fosfodiatilcolina al interior de la vía biliar. La presencia de este fosfolípido en la bilis impide la acción detergente de los ácidos biliares sobre el epitelio de los conductos, la disminución o ausencia de fosfolípidos da origen a una colangitis que evoluciona en los primeros años de vida hacia una fibrosis hepáti$\mathrm{ca}^{10}$, cuya manifestación clínica es el aumento de tamaño y consistencia del hígado, además de alteraciones de las pruebas funcionales hepáticas (ALT, AST, GGT). A diferencia de otras colestasias, en la CIFP-3, el prurito no está presente o es tenue. Por esta razón, sin un examen semiológico acucioso y sin exámenes de laboratorio, que permitan un diagnóstico oportuno, estos pacientes llegan a la edad de adolescentes o adultos jóvenes a consultar por fibrosis o cirrosis hepática con sus complicaciones correspondientes.

En el caso 1 sólo el perfil bioquímico solicitado por su pediatra llamó la atención por el aumento de la AST, posteriormente se completó el estudio concluyendo que el diagnóstico correspondía a una CIFP-3 que estaba presente en tres de los 5 hijo de esta familia. En los tres hermanos afectados se inició tratamiento con ácido ursodeoxicólico $30 \mathrm{mg} / \mathrm{kg}$ de peso al día. El mecanismo de acción de estas sales biliares incluye: impedimeto a la reabsorción de los ácidos biliares a nivel del intestino, protección de la membrana del epitelio canalicular del efecto tóxico que sobre ella producen los ácidos biliares hidrofóbicos, inducción de hipercoleresis, modulación de la secreción de citoquinas e inmunoglobulinas, activación de la función excretora de los hepatocitos de la secreción biliar, y aumento en la excreción de los ácidos biliares endógenos, disminuyendo así el efecto tóxico en los hepatocitos ${ }^{11}$.

El diagnóstico precoz de estas colestasias intrahepáticas, especialmente la CIFP-3, la cuál es asintomática en los primeros años de vida, permite una mejor respuesta al tratamiento con ácido ursodeoxicólico. La pesquisa de una hepatomegalia asintomática, aumento de las aminotransferasas y de la GGT debe encauzar el estudio a descartar esta patología. La investigación debe incluir la biopsia hepática, donde se describe daño y proliferación de los conductillos hepáticos y signo de colestasia mínima, con permeabilidad de la vía biliar. La investigación genética no está disponible en nuestro país. Las muestras de ADN y posteriormente de sangre congelada enviadas a EE.UU. llegaron en malas condiciones, por lo tanto, no contamos con el estudio genético correspondiente. Actualmente, se está utilizando la técnica de inmunotinción de MDR3 en tejido hepático ${ }^{12}$; en estos pacientes esta proteína está disminuida o ausente. El defecto genético se localiza en el cromosoma 7q21. El estudio genético del MDR3 puede detectar diferentes mutaciones. Jacquemin E. y col${ }^{4}$, investigaron 31 pacientes 
con fenotipo de CIFP-3, el estudio reveló 16 mutaciones diferentes en 17 pacientes. Estas alteraciones se encontraron en los 2 alelos en 12 pacientes y sólo en un alelo en los otros 5 . Seis de las mutaciones correspondían al tipo de proteína truncada, en estos pacientes no se detecta la glicoproteína MDR3 por el método de inmunotinción debido a que ésta se destruye rápidamente después de ser sintetizada. Diez mutaciones eran del tipo missence y 2 del tipo nonsense. Los pacientes con mutación tipo missense, donde el MDR3 mantiene algo de actividad transportadora de fosfolípidos, tienen una enfermedad menos severa y responden bien al tratamiento con ácido ursodeoxicólico. No sucede lo mismo en aquellos pacientes en que la mutación se manifiesta por una proteína truncada, donde no hay actividad transportadora de fosfolípidos, permitiendo así que la acción detergente de los ácidos biliares dañe el epitelio de los conductos biliares; en esta situación, la respuesta al ácido ursodeoxicólico es nula o mínima evolucionan con daño hepático progresivo hacia una cirrosis. Las mutaciones heterocigotas de MDR3 son causantes de otras patologías como la colestasia del embarazo y de litiasis de la vesícula biliar ${ }^{12,13}$.

La técnica de inmunotinción en estos pacientes, es variable, pude ser normal o disminuida la presencia de MDR3 en los canalículos biliares de los pacientes con mutación missense, en los que presentan el gen truncado la inmunotinción es negativa. Existen otros tipos de mutación localizada en diferentes segmentos del cromosoma 7 en las que la inmunotinción es normal, pero probablemente el MDR3 identificado carece de actividad transportadora ${ }^{14}$. Por esta razón la técnica de inmunotinción es de poco valor en el diagnóstico de esta enferme$\operatorname{dad}^{15}$. La medición de los fosfolípidos es otra técnica de diagnóstico de las CIFP-3, éstos se encuentran en una concentración muy baja representando apenas el 7\% de los lípidos de la bilis (normal $=20 \%$ ).

Como se trata de un problema genético, puede estar presente en los hermanos, como sucedió en esta familia, por lo cuál es necesario investigarlos a todos con exámenes periódicos de ALT, AST, GGT, con el propósito de llegar al diagnóstico en una etapa temprana de la vida. Si se dispone del estudio genético, éste permitirá establecer precozmente el diagnóstico. Ante la imposibilidad de realizar esta técnica se puede estudiar la concentración de fosfolípidos en la bilis; esto permitirá establecer el diagnóstico antes de que aparezcan manifestaciones de daño de la función hepática, lo que permitirá iniciar precozmente el tratamiento con ácido ursodeoxicólico, previniendo así el daño hepático descrito.

\section{Referencias}

1.- Harris M, Le Couteur, Arias J: Progressive familial Intrahepatic Cholestasis: Genetic Disorders of Biliary Trransporters. J. Gastrol and Hepatol 2005; 20: $807-$ 17.

2.- Wagner M, Trauner M: Transcriptional regulation of Hepatology Transport Systems in Health and Disease: Implication for a Rational Approch to the Treatment of Intrahepatic Cholestasis. A Hepatol 2005; 4: 7799.

3.- De Vree JM, Jacquemin E, Sturn E, et al: Mutation in the MDR3 Gene Cause Progressive Familial Intrahepatic Cholestis. Proc Natl Acad Sci USA 1998; 95: 282-7.

4.- Jacquemin E, De Vree JM, Cresteil D, et al: The Wide Spectrum of Multidrug Resistant 3 Deficiency: From Neonatal Cholestasis to Chirrhosis of Adulthood. Gastroenterology 2001; 120: 1448-58.

5.- Stiehl A, et al: Biliary Decretion of Bile Acids and Lipids in Primary Sclerosis Cholangitis. Influence of Cholestasis and Effect of Ursodeoxycholic Acid Treatment. Hepatology 1995; 23: 283-9.

6.- Jacquemin E, et al: Ursodeoxycholic Acid Therapy in Pediatric Patients with Progressive Familial Intrahepatic Cholestasis. Hepatology 1997; 25: 519-23.

7.- Bull LN, Carlton VE, Starcker ML, et al: Genetic and Morphological Findings in Progressive Familial Intrahepatic Cholestasis (Byler Disease (PFCI-1) and Byler Syndrome): Evidence for Heterogenity. Hepatology 1997; 26: 155-64.

8.- Kalicinski $P$, et al: Surgical Treatment of Progressive Intrahepatic Cholestasis: Comparison of Partial External Biliary Diversion and Ilial Bypass. Eur J Pediatr Surgery 2003; 307-11.

9.- Kurbegov AC, et al: Biliary Diversion for Progressive Familial Intrahepatic Cholestasis: Improved Liver Morphology and Bile Acid Profile. Gastroenterology 
2003; 125: 1227-34.

10.- Smit J, Schinkel A, et al: Homozygous Disruption of the Murine mdr-2P- Glychoprotein Gene Leads to a Complete Absence of Phospholipid from Bile and to a Liver Disease. Cell 1993; 75: 451-62.

11- Beuers $U$, et al: Mechanism of Action of Urso Deoxycholic Acid. Falk Symposium 102, October 1997: 262-7. Kluwer Academic Publishers, Boston.

12.- Keitel V, Burdelski M, Warskulat U, et al: Expression and Localization of Hepatobiliary Transport Proteins in Progressive Familial Intrahepatic Cholestasis. Hepatology 2005; 41 (5): 1160-72.

13.- Rosmorduc O, Hermelin B, Paupon R: MDR3 Gene Defect in Adults with symptomatic Inntrahepatic and Gallbladder Cholesterol Cholelitiasis. Gastroenterology 2001; 120: 1459-67.

14.- Jacquemin E, Cresteil D, Manouvrier $S$, et al: Heterozygous Non- sese Mutation of the MDR3 Gene in Familial Intrahepatic Cholestasis of Pregnancy. Lancet 1999; 353: 210-1. 